\title{
Assesment and interpretation of negative forelimb allometry in the evolution of non- avian Theropoda
}

\author{
José A. Palma Liberona ${ }^{*}$, Sergio Soto-Acuña ${ }^{1}$, Marco A. Mendez ${ }^{2}$ and Alexander O. Vargas ${ }^{*}$
}

\begin{abstract}
Background: The origin of birds is marked by a significant decrease in body size along with an increase in relative forelimb size. However, before the evolution of flight, both traits may have already been related: It has been proposed that an evolutionary trend of negative forelimb allometry existed in non-avian Theropoda, such that larger species often have relatively shorter forelimbs. Nevertheless, several exceptions exist, calling for rigorous phylogenetic statistical testing.

Results: Here, we re-assessed allometric patterns in the evolution of non-avian theropods, for the first time taking into account the non-independence among related species due to shared evolutionary history.

We confirmed a main evolutionary trend of negative forelimb allometry for non-avian Theropoda, but also found support that some specific subclades (Coelophysoidea, Ornithomimosauria, and Oviraptorosauria) exhibit allometric trends that are closer to isometry, losing the ancestral negative forelimb allometry present in Theropoda as a whole.

Conclusions: Explanations for negative forelimb allometry in the evolution of non-avian theropods have not been discussed, yet evolutionary allometric trends often reflect ontogenetic allometries, which suggests negative allometry of the forelimb in the ontogeny of most non-avian theropods. In modern birds, allometric growth of the limbs is related to locomotor and behavioral changes along ontogeny. After reviewing the evidence for such changes during the ontogeny of non-avian dinosaurs, we propose that proportionally longer arms of juveniles became adult traits in the small-sized and paedomorphic Aves.
\end{abstract}

Keywords: Theropoda, Allometry, Evolution, Phylogeny, Ontogeny, Paedomorphosis

\section{Background}

Along the theropod-bird transition, many functional and morphological transformations took place, including a potential for flight, which is the focal point of several studies (e.g. references [1-5] among others). Two key morphological changes required for flight were the reduction of body size $[6,7]$ and the increase in relative forelimb size $[8,9]$. However, both of these changes had already begun in non-avian Theropoda, long before the origin of avian flight. Further, they were likely related to each other: An evolutionary trend of negative allometry appears to have existed in non-avian theropods, such that species with a larger body size often have proportionally

\footnotetext{
* Correspondence: jpalmaliberona@gmail.com; alexvargas@uchile.cl ${ }^{1}$ Laboratorio de Ontogenia y Filogenia, Departamento de Biología, Facultad de Ciencias, Universidad de Chile., Las Palmeras 3425, Santiago, Chile Full list of author information is available at the end of the article
}

smaller forelimbs $[10,11]$. Nevertheless, it is clear this was not always the case: some large-sized species have proportionally larger arms, while other small-sized species have proportionally smaller arms. Based on such observations, a lack of negative allometry has been suggested within Therizinosauria [11] and Ornithomimosauria [10]. Additionally, Aves evolved an opposite trend of positive allometry (proportionally larger arms in larger species) that is already present among Mesozoic taxa [11], and continues in modern Neornithes [12]. The evidence for a diversity of evolutionary trends opens the question of whether the data indeed supports the existence of a main trend of negative forelimb allometry for non-avian theropods. This question should be approached through phylogenetic statistical methods, that properly consider the non-independence of data from related specimens when estimating allometric coefficients. It has been argued that incorrect branch

(c) The Author(s). 2019 Open Access This article is distributed under the terms of the Creative Commons Attribution 4.0 International License (http://creativecommons.org/licenses/by/4.0/), which permits unrestricted use, distribution, and 
lengths can have an effect on Phylogenetic Independent Contrasts, therefore raw regression values have been used and then compared with ancestral node reconstruction values to argue that phylogeny did not overly affect estimated parameters [11]. However, without correcting or taking into account the covariation of non-independent data, regressions may incur in bias for the estimated parameters as well as elevated type I errors [13].

Here, we have re-assessed the evidence for evolutionary allometric trends of the forelimb in non-avian theropods, using the largest dataset to date, new combinations of statistical tools, and an assessment of how specific subclades within Theropoda. may have evolved different evolutionary trends. Our results confirm negative allometry as the main trend for non-avian Theropoda, and allow us to formally identify those theropod subclades that likely had a different trend. To explain evolutionary allometric trends, we discuss how these are related to the allometric growth of the limbs in the ontogeny of modern birds, and these in turn to changes in behavior and locomotion along development. We review the evidence for forelimb function and allometry in the ontogeny of non-avian dinosaurs, and the potential relation of body size reduction, paedomorphosis, and forelimb size near the origin of birds.

\section{Materials and methods}

\section{Measurements}

We collected measurements of 163 fossil specimens within Theropoda across 108 genera (Additional file 2), including species represented by multiple specimens. We aimed to identify the allometric trends that existed previous to flight specialization and positive allometric trends of Aves; therefore, no avian taxa were included, considering Aves as the clade of volant theropods containing the last common ancestor of Archaeopteryx lithographica and Vultur gryphus, and all its descendants [14]. This approach was conservative in that we still included several fossil taxa that might have been volant, and that are placed within Aves by some authors, namely Anchiornis huxleyi, Aurornis xui (proposed as a synonym of Anchiornis in [15]), Xiaotingia zhengi, Eosinopteryx brevipenna and Serikornis sungei. These taxa were labelled as "Anchiornis-related taxa" in this study. We also included Scansoriopterygidae, which have been placed within Aves by some authors [7], but also in a non-avian position (within Avialae in [16]; and as the sister clade of Oviraptorosauria in [17]). Likewise, we did not exclude Troodontidae or Dromeosauridae, despite containing potentially volant forms, and despite proposed phylogenetic positions that would place them within Aves as defined above $[18,19]$.

Measurements of femoral and humeral length (FL and HL respectively) were collected from original published descriptions and studies confirming that humerus and femur were well preserved and non-controversial. This likely introduced some variation due to possible methodological differences in the way measurements were taken by different authors, but also allowed for a dataset of worldwide specimens that is much larger than could be afforded given the restrictions in time and resources that are required for taking all measurements in person. FL was selected given that it is a good proxy for body size [7, 20-22] and is conserved and reported more often than other measurements such as snout-vent length. Therefore, even though FL can be subject to specific evolution (decoupling from body size variation [11]), it allowed us to maximize the number of specimens in our dataset. We chose HL over other forelimb elements since the humerus is most often conserved and reported, even though other elements may potentially show stronger negative allometry [23]. Following standard procedure, all collected measurements were $\log 10$ transformed allowing us to fit our data using linear models instead of power law equations. The complete dataset can be found in the supporting online information (Additional file 2).

Our dataset included all specimens, regardless of ontogenetic stage, and regardless of whether some species were represented by multiple individuals. One concern that arises is whether species with multiple individuals may be over-represented and generate bias. Although phylogenetic methods can arguably address this problem [24], we also tested this question by producing a second reduced dataset, with only one specimen per species, which was selected as the single largest specimen of that species. Another possible concern is that adults and juveniles should not be considered together in the same analysis, in order to properly distinguish evolutionary trends from ontogenetic trends (regardless of whether they may be related). To test if mixed ontogenetic status produced any significant differences, we generated a dataset comprised only by adults, in order to observe "pure" evolutionary trends. We considered a juvenile to be a young animal with no signs of impending maturity that would place it as an adult or sub-adult (following [25]).This "adult" dataset was maximally conservative, in that we excluded every specimen that has been proposed to be a juvenile, without independent assessment of ontogenetic status or standing controversies (which are common [25];). We also generated a dataset composed only of specimens proposed to be juveniles, to allow a preliminary examination of any evidence that juveniles may have had a different allometric trend.

\section{Phylogenetic relationships}

Although the main phylogenetic relationships within Theropoda are mostly agreed upon, some aspects are still under discussion, leading to phylogenetic uncertainties (unsolved 
polytomies) that have an effect on parameter estimation [26-29]. For this study, we created 6 topologies as informal supertrees. The polytomies present on these topologies were then stochastically resolved and their branch lengths calibrated using a stochastic sampling method (Fig. 1) generating 1000 trees per topology with variations in topology and branch lengths.

The six supertrees used were constructed using Mesquite v3.4 [30] (Fig. 1a). We labeled these topologies with numbers 1 through 6, these are: 1) considering Megaraptora closer to Allosaurus than to Tyrannosaurus [31], with Troodontidae and Scansoriopterygidae closer to Aves than Dromaeosauridae, while Anchiornis and related taxa (Xiaotingia, Aurornis, Eosinopteryx and Serikornis) are closer to Aves than to Troodontidae [7]; 2) similar to topology 1 but

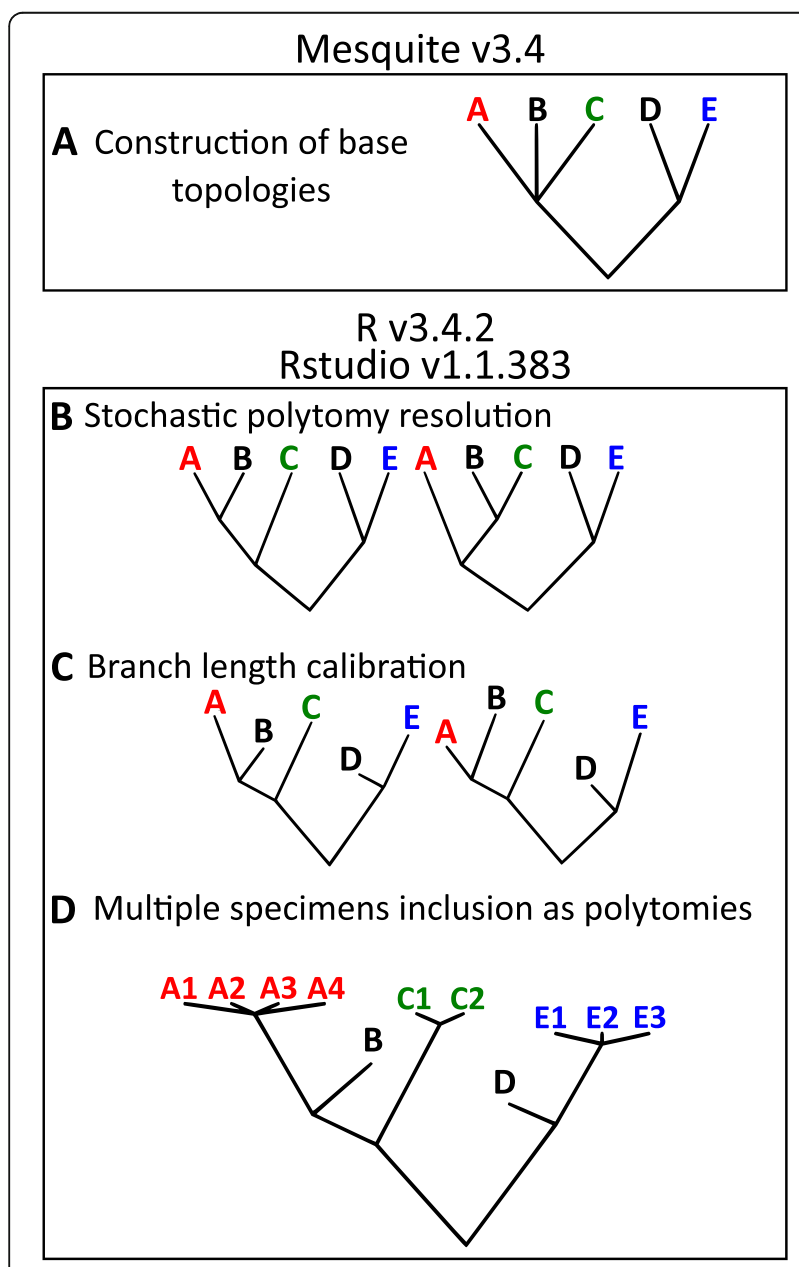

Fig. 1 Tree construction and calibration workflow. a base topologies are manually constructed in Mesquite, $\mathbf{b}$ base topologies are exported to RStudio and their polytomies stochastically resolved, c Branch lengths are calibrated using species age, 1000 time calibrated trees are generated for each topology in steps (b) and (c, d) branch tips of species represented by multiple specimens are replaced with a branch length 0 polytomy in order to account for intraspecific variation considering a clade Megaraptora closer to Tyrannosaurus than to Allosaurus [32, 33]; 3) placing Megaraptora closer to Allosaurus than to Tyrannosaurus, Dromaeosauridae and Troodontidae closer to each other than either is to Aves, and Scansoriopterygidae as the sister group to Aves [16]; 4) similar to topology 3 but with Megaraptora closer to Tyrannosaurus than to Allosaurus; 5) considering Megaraptora as closer to Allosaurus than to Tyrannosaurus; Dromaeosauridae closer to Aves than Troodontidae and Scansoriopterygidae as the sister taxa of Oviraptorosauria [17]; and 6) similar to topology 5 but placing Megaraptora closer to Tyrannosaurus than to Allosaurus. In order to maximize the species represented in the topologies we used a similar method to that described in [29] inserting those species absent from published phylogenies in the most resolved non-controversial polytomy. The constructed supertrees are shown in the supporting information (Additional file 1: Figure S3).

The six topologies were exported to RStudio v1.1.383 [34] running as an IDE for R v3.4.2 [35], with all polytomies randomly dichotomized using the function multi2di from the R package ape v4.1 [36] (Fig. 1b). Branch lengths were calibrated using the reported geological age of each specimen in the corresponding publication or the compiled geological ages available in the Paleobiology Database (PaleoDB, https://paleobiodb.org/). If no data on geological age was available for a given specimen, we used either the sister taxon's age or the complete clade age range (see supplementary methods in the Additional file 1). These age ranges were then used in the cal3 algorithm of the R package paleotree v2.7 [37-39] (Fig. 1c). This algorithm calibrates branch lengths using a stochastic sampling of node ages under a birth-death-sampling model; for this we used the theropod sampling rate reported in [40] and used it to estimate the extinction rate. Diversification rate was assumed to be equal to the extinction rate, which has been argued to be a reasonable assumption for extinct clades [39]. After dichotomization and calibration 1 was added to each branch length in order to maintain the node structure derived from the base topologies. The use of geological age for branch calibration is a common methodological approach when dealing with data from the fossil record [22, 41-43], although there are many ways in which this method can create bias as a result of missing information. However, our results did not show significant changes when using a "null" model of uniform branch length (not shown), showing them to be robust to branch length variation.

For those analyses considering the complete dataset, including multiple specimens per species, we adapted the method described in [44] for Phylogenetically Independent Contrasts (PIC) and used it for Phylogenetic Generalized Least Squares (PGLS) [45], looking to maximize the shared evolutionary history represented in the trees for 
conspecifics, and thus minimizing their relative weight [46, 47]. Therefore, we replaced the tips of each species represented by more than a single specimen with a zero-branch length polytomy (Fig. 1d). However, it should be noted that this method assumes that every specimen of a species has the same geological age and ignores possible factors that may reduce the covariance between specimens.

\section{Regressions and statistical tests}

Regressions were done using PGLS [48] under two different evolutionary models: Brownian Motion (BM) [49, 50] (reported as a model for body size evolution in Theropoda [21]); as well as Ornstein-Uhlenbeck (OU) [51, 52]. However, we advise caution when interpreting results with the latter, since it has been reported to incur in elevated type I errors when used for low sample sizes or low attraction strength $(\alpha)$ [52]. Regressions using BM were done with simultaneous estimation of phylogenetic signal using Pagel's $\lambda[46,53,54]$, which is a coefficient that downweighs the covariance matrix derived of the phylogenetic trees, according to how closely does the observed trait distribution resemble one simulated under pure Brownian Motion (BM). In this sense a $\lambda=0$ implies a trait distribution completely independent of the phylogeny and $\lambda=1$ one that exactly mirrors what would be expected had the trait evolved under BM. For the regressions we used the $\mathrm{R}$ packages nlme v3.1 [55] and ape v4.1 [36]. When $\lambda$ estimation was not possible a fixed value of $\lambda=1$ was assigned and an additional regression using Ordinary Least Squares (OLS) was done, which is the equivalent of performing a PGLS with $\lambda=0$, in order to assess the full range of possible values. We performed the regressions on each of the 6000 dichotomous and calibrated trees (1000 per topology) and then, the estimated parameters were pooled using multiple imputation methods $[56,57]$ for each evolutionary model. It is worth noting that these methods converge to the one presented in [58] when extended to all the possible trees. Parameter pooling was done using the R package mice v2.30 [59] and pooled parameters were estimated for each topology plus a total pool considering all 6000 regressions. We also performed regressions for each clade within Theropoda using PGLS in order to evaluate clade-specific allometric patterns. All these analyses were carried out twice for each model: once using the complete dataset (including intraspecific variability) and once with the reduced dataset (including only a single largest specimen per species, see Additional file 1: Tables S5 for BM and S14 for OU). We also used PGLS to perform regressions on the dataset of Theropoda that excluded juvenile specimens, as well as the dataset composed only by specimens proposed to be juveniles.

To test whether any specific subclades significantly deviate from the main allometric trend of Theropoda, we used Phylogenetic ANCOVA (PANCOVA) [60]. Starting with the complete dataset, we tested for differences in Scansoriopterygidae, Troodontidae, Dromaeosauridae, Oviraptorosauria, Therizinosauria, Ornithomimosauria, Compsognathidae, Tyrannosauroidea, Megaraptora, Megalosauroidea, Ceratosauria, Coelophysoidea and the paraphyletic Anchiornis-related taxa. However, because most of these subclades are represented by few specimens, results must be considered as a first approach to identifying those sublcades that are most likely to show a different trend. PANCOVA tests were performed over a subset of 200 trees for each topology due to computational limitations, and the resulting $p$-values were then pooled using a meta-analysis method for non-independent tests [61]. Taxa consistently showing significant deviations from the main allometric trend for Theropoda across all topologies were then excluded and a new set of regressions were carried out. This process of regressions and PANCOVA testing was repeated in an iterative fashion until no new taxa were consistently excluded.

\section{Results}

\section{Main Allometric trends and outliers}

Our general analysis (complete dataset of all Theropoda) shows negative forelimb allometry that is significantly different from isometry for both $\mathrm{BM}$ and OU models (BM: 0.914, 95\% CI [0.864, 0.964], $\lambda=0.930$, Fig. 2a; OU: $0.941,95 \%$ CI $[0.900,0.982], \alpha=0.013$, Additional file 1: Figure S1). The estimated parameters for the reduced dataset (with only one specimen per species) also showed negative forelimb allometry that is significantly different from isometry (BM: 0.901, 95\% CI [0.843, 0.959], $\lambda=0.954$; OU: $0.906,95 \%$ CI [0.848, 0.965], $\alpha=$ $0.013)$. It should be noted that no statistically significant difference was found between the allometric coefficients estimated for the complete and reduced dataset. Thus, we discarded any concerns about over-representation of species with multiple specimens. Allometric coefficients and $\lambda$ or $\alpha$ estimations for the pooled regressions of topologies 1 through 6 resulted in similar values for BM and OU respectively (Additional file 1: Tables S1 and S2 for BM, Tables S7 and S8 for OU), therefore the total pooled coefficient and $\lambda$ or $\alpha$, considering all 6000 trees across the six topologies, properly represents the mean estimated parameters for all the considered phylogenetic hypotheses.

Estimated parameters for the dataset comprising only adult specimens (BM: $0.877,95 \%$ CI $[0.808,0.945], \lambda=$ 0.944; OU: $0.877,95 \%$ CI $[0.758,0.996], \alpha=0.493)$ showed no statistical difference when compared to those of our general analysis. Also, a dataset comprised only of specimens proposed to be juveniles resulted in similar parameters to adults under BM $(0.885,95 \%$ CI $[0.798,0.973], \lambda=$ 0.949), differing only on their intercepts (Fig. 2b), with 


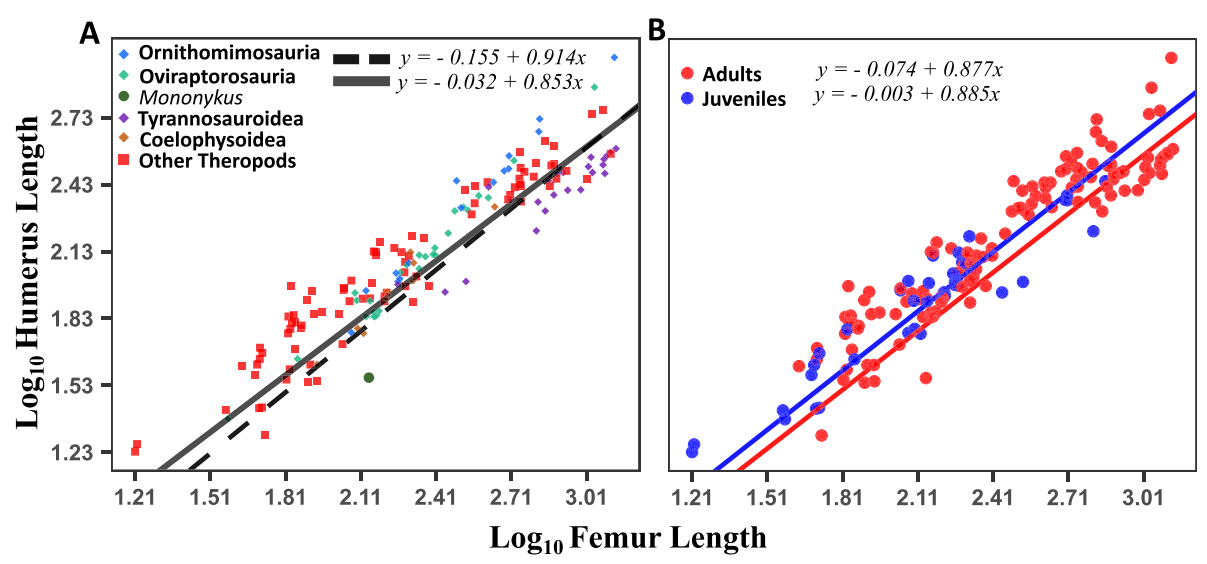

Fig. 2 PGLS regressions under BM for humeral against femoral measurements, (a) regressions for the complete dataset before (dotted line) and after (continuous line) excluding subclades that showed significant differences from the main allometric trend after Phylogenetic ANCOVA testing (Oviraptorosauria, Ornithomimosauria, Tyrannosauroidea, Coelophysoidea and Mononykus), (b) regressions for adult (red) and juvenile (blue) specimens

higher differences found under OU $(0.938,95 \% \mathrm{CI}[0.839$, 1.037], $\alpha=0.007$ ). However, under OU, low $\alpha$ values and low sample size (37 specimens) might make these parameters unreliable [52]. Therefore, we found no evidence to exclude juvenile specimens.

Upon using PANCOVA to test for clade-specific deviations from the main trend of negative forelimb allometry, we found that Oviraptorosauria, Ornithomimosauria, and Coelophysoidea showed significant differences consistently across all topologies under both $\mathrm{BM}$ and $\mathrm{OU}$, with the addition of Tyrannosauroidea only under BM (Additional file 1: Table S3) and Scansoriopterygidae and Megalosauroidea only under OU (Additional file 1: Tables S9 and S10). We then carried out a new analysis with all these subclades excluded, to test whether any additional subclades deviate from the main trend. We also decided to exclude the alvarezsaurid Mononykus olecranus, a small-sized specimen with short forelimbs, whose position in charts is clearly offset from the distribution of most specimens (Fig. 2a). Although testing through phylogenetic ANCOVA cannot be carried out for a single data point, it is very likely that Mononykus does not conform to the main trend of negative allometry, not only because of its proportionally small forelimbs, but also because Mononykus evolved unique and extreme morphofunctional specialization of the forelimb, unlike any other theropods in our dataset (including its closest relative Haplocheirus). Mononykus has short, robust and highly muscularized forelimbs, and a monodactyl hand presenting a uniquely enlarged digit and claw. Along with a short radius and ulna and large olecranon process, the short and stout humerus has large muscle insertion sites, associated to a keeled and ossified sternum. This makes the forelimbs functionally comparable to those of digging tetrapods, which combined with the toothless and very slender snout suggest specialization in feeding on small insects [62].
Regressions on the remaining theropods resulted in lower allometric coefficients for both the complete (Fig. 2a) and reduced datasets, resulting in similar values for all topologies under BM (Table 1) and OU (Additional file 1: Table S12) with all allometric coefficients showing negative allometry and significant difference from isometry. After retesting for deviations from the main forelimb allometric trend on the remaining subclades using PANCOVA, some additional taxa showed significant differences, but this depended on the topology used: No taxa presented significant differences across all six topologies (Additional file 1: Table S4 for BM and supplementary Table S11 for OU). Our analysis did not consistently support a different trend for Therizinosauria, which had been suggested to be outliers [11].

\section{Allometric trends within Theropod subclades}

PGLS regressions for each clade largely confirmed the results obtained through PANCOVA: allometric coefficients estimated for Coelophysoidea, Ornithomimosauria and Oviraptorosauria resulted in isometry or values very near it, and no significant difference was observed with the reduced dataset, discarding the possibility that these trends could be due to overrepresentation of any single species (Table 2, Additional file 1: Table S5 for BM and supplementary Tables S13 and S14 for OU). For Oviraptorosauria the allometric coefficient recovered was similar to that reported in previous studies $(1.014$ for 12 specimens [63]).

In Tyrannosauroidea, a clade that was also found to be significantly different under BM, there is a lower value of negative allometry than the main allometric trend for Theropoda, but a wide confidence interval, with no significant difference from isometry. Given that the allometric coefficient of Tyrannosauroidea is within what 
Table 1 Linear regression values for forelimb allometry for Theropoda under BM after excluding Oviraptorosauria, Ornithomimosauria, Tyrannosauroidea and Coelophysoidea

\begin{tabular}{|c|c|c|c|c|c|c|c|c|}
\hline \multicolumn{5}{|c|}{ Complete dataset } & \multicolumn{4}{|c|}{ Reduced dataset } \\
\hline Topology & Intercept & Slope & Slope $95 \%$ Cl & $\lambda$ & Intercept & Slope & Slope $95 \% \mathrm{Cl}$ & $\lambda$ \\
\hline 1 & 0.026 & 0.859 & $(0.797,0.922)$ & 0.939 & 0.079 & 0.840 & $(0.772,0.908)$ & 0.892 \\
\hline 2 & 0.011 & 0.862 & $(0.800,0.923)$ & 0.939 & 0.068 & 0.840 & $(0.773,0.907)$ & 0.877 \\
\hline 3 & 0.035 & 0.849 & $(0.787,0.912)$ & 0.938 & 0.092 & 0.831 & $(0.763,0.899)$ & 0.878 \\
\hline 4 & 0.019 & 0.851 & $(0.789,0.914$ & 0.936 & 0.081 & 0.831 & $(0.764,0.898)$ & 0.860 \\
\hline 5 & 0.057 & 0.848 & $(0.784,0.911)$ & 0.937 & 0.115 & 0.827 & $(0.761,0.893)$ & 0.875 \\
\hline 6 & 0.043 & 0.849 & $(0.785,0.913)$ & 0.936 & 0.108 & 0.827 & $(0.762,0.892)$ & 0.848 \\
\hline $1-6$ & 0.032 & 0.853 & $(0.789,0.917)$ & 0.938 & 0.091 & 0.8336 & $(0.765,0.900)$ & 0.872 \\
\hline
\end{tabular}

Intercept, Slope and Slope $95 \% \mathrm{Cl}$ are estimations obtained after pooling 1000 dichotomous time-scaled trees generated for the specified topology or topologies. $\lambda$ is a simple mean of Pagel's $\lambda$ estimations on the same regressions

could be expected for other theropods, we performed a new PANCOVA in order to test if this clade was initially shown to be significantly different due to the influence of Coelophysoidea, Ornithomimosauria and Oviraptorosauria pulling the main theropod allometric coefficient towards higher values. The results of this test once again showed significant differences between Tyrannosauroidea and the rest of the dataset (Additional file 1: Table S6).

The case of Scansoriopterygidae is peculiar: its allometric coefficient indicates isometry, however, the confidence interval is so wide that it doesn't allow us to rule out either strong negative or positive allometries (Table 2, Fig. 3, Additional file 1: Tables S5. S13 and S14). Similarly, wide confidence intervals for allometric coefficients are found in Troodontidae, Compsognathidae and the subclade containing Allosauroidea and Megaraptoridae (only possible in topologies 1,3 and 5), although in these subclades, the upper limit of the confidence interval is near isometric, rather than one of positive allometry (Table 2. Figure 3, Additional file 1: Tables S5, S13 and S14). Wide confidence intervals in Scansoripterygidae could result from the very low sample size, although other subclades with similar sample sizes such as Therizinosauria and Megalosauroidea show narrower confidence intervals.

In subclades that are proposed as close relatives of Aves, we evaluated the allometric coefficient of Anchiornis-related taxa, Troodontidae, and Dromaeosauridae, as well as combinations of these taxa (Dromaeosauridae + Troodontidae for all topologies and Troodontidae + Anchiornis-related taxa in topologies 1-4). Scansoriopterygidae was considered, although some authors have argued they are not among the closest relatives of Aves [17]. The Anchiornis-related taxa and Troodontidae show coefficients of negative allometry, but these are not significantly different from isometry. However, when grouped together (topologies 1-2 as a paraphyletic group and topologies 3-4 as a monophyletic clade) the resulting allometric coefficients of this clade showed strong negative allometry, significantly different from isometry.
Dromaeosauridae showed strong negative allometry under BM both alone and when grouped with Troodontidae, being significantly different from isometry in all cases. Similar values were found after removing the Microraptor specimens, due to them being possible outliers, given their flight adaptations (Table 2). Under OU Dromaeosauridae showed wider confidence intervals, where the upper limit reaches isometry (Additional file 1: Tables S13 and S14).

\section{Discussion}

As assessed from the total dataset (without excluding any outliers), we confirm support for a main trend of negative forelimb allometry in the evolution of nonavian Theropoda $[10,11]$. By testing for the presence of outliers, we have also provided formal support for the notion that some subclades likely presented a different trend in arm size evolution. However, upon excluding these outliers, further rounds of PANCOVA testing did not consistently identify any additional subclades as outliers. Had we continued until most subclades were separated, this would have supported a scenario of "total diversity", where each clade has its own distinctive trend. Instead, our data suggests a scenario in which negative allometry represents the primitive or "default" trend for Theropoda, and subclades with different trends ("outliers") are derived (see below for further discussion on the basal Coelophysoidea). This is consistent with the main trend recovered for the complete dataset, and the observation that larger animals with proportionally smaller forelimbs are present across several subclades.

Although the evolutionary trend of negative allometry has been discussed by some authors, these have offered few explanations about its possible underlying causes. The existence of such a well-defined macroevolutionary trend defies explanation of forelimb proportions as multiple independent events of adaptation. In this regard, allometric trends in evolution often reflect ontogenetic allometries, such that evolutionary changes in adult body 
Table 2 Pooled linear regressions under BM using the complete dataset for specific subclades

\begin{tabular}{|c|c|c|c|c|c|c|}
\hline Clade & $\mathrm{N}$ & Top. & Intercept & Slope & Slope $95 \% \mathrm{Cl}$ & $\lambda$ \\
\hline \multirow[t]{2}{*}{ Scansoriopterygidae } & 4 & $1-6$ & -0.053 & 1.073 & $(0.741,1.404)$ & $1^{a}$ \\
\hline & & - & -0.075 & 1.085 & $(0.527,1.644)$ & $0^{a}$ \\
\hline \multirow[t]{3}{*}{ Troodontidae + Anchiornis related } & 20 & $1-2$ & 0.356 & 0.742 & $(0.520,0.964)$ & $1^{\mathrm{a}}$ \\
\hline & & $3-4$ & 0.352 & 0.743 & $(0.521,0.964)$ & $1^{\mathrm{a}}$ \\
\hline & & - & 0.685 & 0.577 & $(0.380,0.774)$ & $0^{\mathrm{a}}$ \\
\hline \multirow[t]{2}{*}{ Anchiornis related } & 11 & $1-6$ & 0.067 & 0.928 & $(0.723,1.132)$ & $1^{a}$ \\
\hline & & - & 0.025 & 0.962 & $(0.659,1.264)$ & $0^{a}$ \\
\hline \multirow[t]{3}{*}{ Dromaeosauridae+ Troodontidae } & 31 & $1-2$ & 0.144 & 0.833 & $(0.691,0.975)$ & 0.813 \\
\hline & & $3-4$ & 0.133 & 0.839 & $(0.695,0.983)$ & 0.813 \\
\hline & & $5-6$ & 0.173 & 0.832 & $(0.692,0.971)$ & 0.819 \\
\hline \multirow[t]{2}{*}{ Troodontidae } & 9 & $1-6$ & 0.300 & 0.739 & $(0.436,1.042)$ & $1^{\mathrm{a}}$ \\
\hline & & - & 0.186 & 0.797 & $(0.479,1.115)$ & $0^{\mathrm{a}}$ \\
\hline \multirow[t]{3}{*}{ Dromaeosauridae } & 22 & $1-4$ & 0.328 & 0.757 & $(0.597,0.917)$ & $1^{\mathrm{a}}$ \\
\hline & & $5-6$ & 0.399 & 0.728 & $(0.567,0.888)$ & $1^{a}$ \\
\hline & & - & 0.279 & 0.775 & $(0.732,0.819)$ & $0^{\mathrm{a}}$ \\
\hline \multirow[t]{3}{*}{ Dromaeosauridae excluding Microraptor } & 15 & $1-4$ & 0.410 & 0.721 & $(0.523,0.919)$ & $1^{\mathrm{a}}$ \\
\hline & & $5-6$ & 0.471 & 0.697 & $(0.503,0.890)$ & $1^{\mathrm{a}}$ \\
\hline & & - & 0.279 & 0.775 & $(0.732,0.819)$ & $0^{\mathrm{a}}$ \\
\hline \multirow[t]{2}{*}{ Oviraptorosauria } & 28 & $1-4$ & -0.204 & 0.988 & $(0.936,1.041)$ & 0.947 \\
\hline & & $5-6$ & -0.189 & 0.987 & $(0.930,1.043)$ & 0.946 \\
\hline \multirow[t]{2}{*}{ Therizinosauria } & 5 & $1-6$ & 0.062 & 0.912 & $(0.755,1.070)$ & $1^{\mathrm{a}}$ \\
\hline & & - & 0.217 & 0.849 & $(0.587,1.111)$ & $0^{a}$ \\
\hline \multirow[t]{2}{*}{ Ornithomimosauria } & 15 & $1-6$ & -0.348 & 1.057 & $(0.928,1.186)$ & $1^{\mathrm{a}}$ \\
\hline & & - & -0.543 & 1.143 & $(1.040,1.245)$ & $0^{\mathrm{a}}$ \\
\hline Compsognathidae & 7 & $1-6$ & -0.042 & 0.876 & $(0.580,1.172)$ & 0.717 \\
\hline Tyrannosauroidea & 23 & $1-6$ & 0.018 & 0.859 & $(0.650,1.068)$ & 0.437 \\
\hline Tyrann. + Megaraptoridae & 25 & $2,4,6$ & -0.0171 & 0.882 & $(0.709,1.054)$ & 0.772 \\
\hline \multirow[t]{2}{*}{ Allo. + Megaraptora } & 8 & $1,3,5$ & 0.424 & 0.719 & $(0.266,1.172)$ & $1^{a}$ \\
\hline & & - & 0.497 & 0.695 & $(0.206,1.184)$ & $0^{a}$ \\
\hline \multirow[t]{2}{*}{ Megalosauroidea } & 5 & $1-6$ & -0.258 & 0.988 & $(0.930,1.046)$ & $1^{a}$ \\
\hline & & - & -0.268 & 0.991 & $(0.909,1.073)$ & $0^{a}$ \\
\hline Ceratosauria & 9 & $1-6$ & 0.276 & 0.763 & $(0.642,0.884)$ & 0.930 \\
\hline \multirow[t]{2}{*}{ Coelophysoidea } & 10 & $1-6$ & -0.463 & 1.073 & $(0.863,1.282)$ & $1^{\mathrm{a}}$ \\
\hline & & - & -0.407 & 1.054 & $(0.842,1.265)$ & $0^{a}$ \\
\hline Non-Maniraptoriform Tetanurae & 44 & $1-6$ & -0.047 & 0.896 & $(0.811,0.980)$ & 0.763 \\
\hline Non-Maniraptoriform Tetanurae excluding Tyrann. & 21 & $1-6$ & -0.055 & 0.908 & $(0.815,1.000)$ & 0.753 \\
\hline
\end{tabular}

$\mathrm{N}$ is the number of specimens, Intercept, Slope and Slope $95 \% \mathrm{Cl}$ are estimations obtained after pooling 1000 dichotomous time-scaled trees generated for the

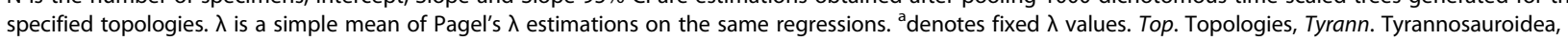
Allo. Allosauroidea

size result in new proportions, as expected according to the pre-existing ontogenetic trends [64-66]. Therefore, an evolutionary trend of negative allometry could reflect a negative allometry in the ontogeny of non-avian theropods, namely, slower growth of the forelimbs [10]. Unlike non-avian theropods, in birds there is an opposite evolutionary trend of positive forelimb allometry, such that larger species have proportionally larger forelimbs [12]. Accordingly, positive allometric growth of the forelimbs has been documented in the ontogeny of several birds $[67,68]$ and has been explicitly discussed for Oceanodroma leucorhoa [69], Larus californicus [70] and 


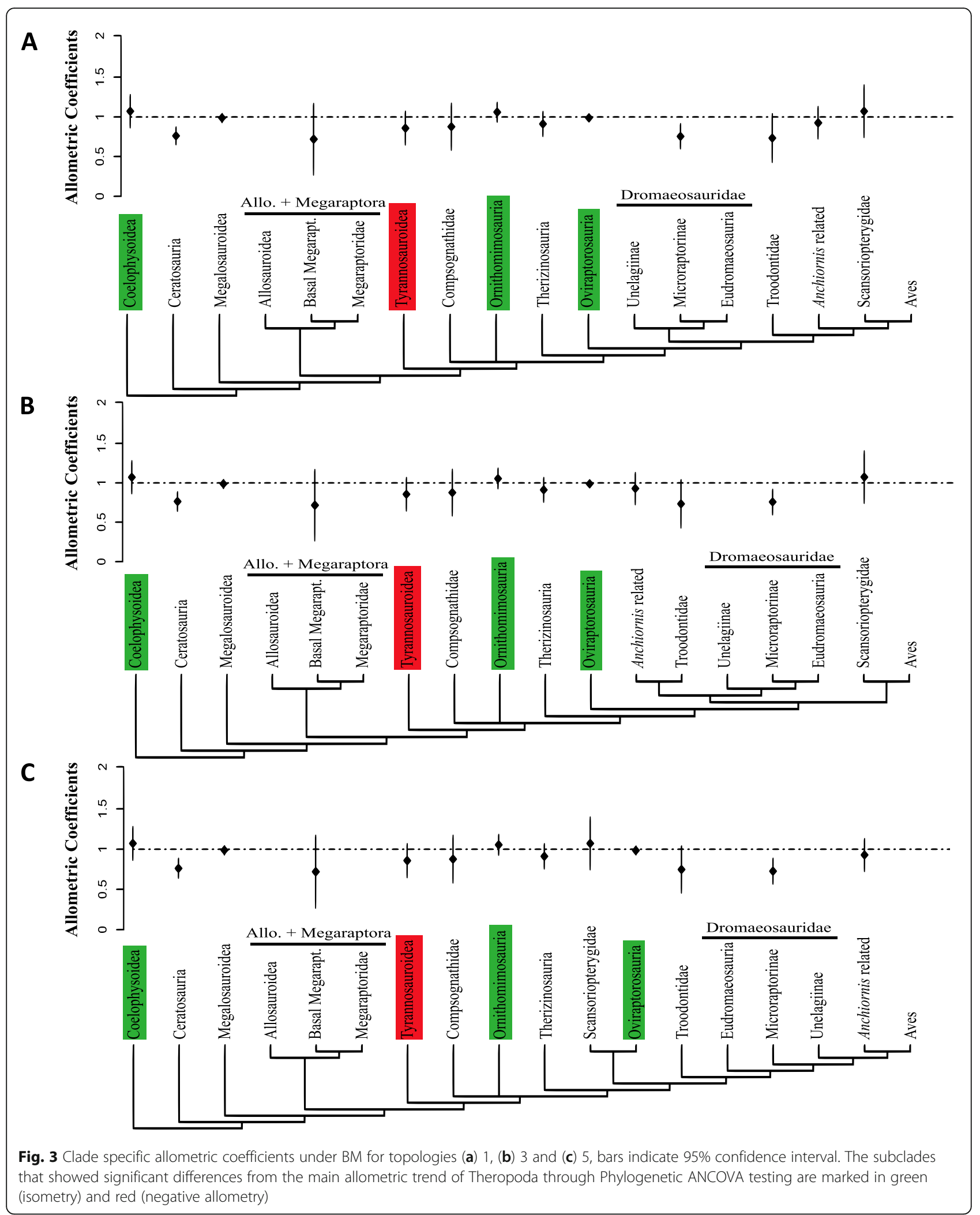


Anous minutus [71]. Modern birds present an ample diversity of relative limb size proportions and locomotor strategies, which can also vary during ontogeny [67]. In some species, the phase of increased forelimb growth occurs at a later ontogenetic stage; for example, in Anas platyrhynchos, negative allometric growth of the forelimb occurs first; forelimb growth only increases at later stages, as forelimbs become relevant in jumping, swimming, landing, and finally flight [72]. Similarly, in Vanellus vanellus, there is a marked delay of forelimb growth during early ontogenetic stages, when juveniles are terrestrial and have well developed hindlimbs. Forelimb growth increases thereafter, as they become involved in flight [73]. In other species such as the Emu Dromaius novahollandiae, hatchlings are born with reduced forelimbs [68], but maintain little activity and growth up to the flightless adult, which presents reduced wings. Based on these observations, we propose that patterns of allometric forelimb growth in modern birds are related to their degree of functional activity (mostly locomotor) at different ontogenetic stages. This suggests that putative negative allometry in the ontogeny of theropod dinosaurs could be related to an important phase of decreased function and activity of the forelimb.

The possibility of slower forelimb growth in the ontogeny of any non-avian dinosaur was first brought up by Gould and Lewontin, who were not satisfied with adaptive explanations for marked forelimb reduction in Tyrannosaurus [74]. Instead, they proposed that forelimb reduction was not an adaptation but "a developmental correlate of allometric fields for relative increase in head and hindlimb size", a morphodynamic view that has been later expanded upon [75]. Slower growth of the forelimb in non-avian theropods is an explicit hypothesis, but fossils of juvenile specimens that can be confidently assigned to a given genus (let alone species) are rare. The best formal attempt has been carried out in Allosaurus, for which large concentrations of disarticulated remains have been found. Numerous femora and humeri representing different ontogenetic stages have been studied using LAGs (Lines of Arrested Growth) to estimate the age of each element. This uncovered negative allometric growth of the humerus [76], as expected within our interpretation. Ontogenetic negative allometry has been argued for humerus length in Tyrannosauridae [77], although this analysis grouped specimens from different genera, and the juvenile status of some specimens has been questioned [78]. No other studies have provided any statistical assessment of negative forelimb allometry in the ontogeny of non-avian theropods. In the data set used in our study, some genera were represented by multiple specimens exhibiting body size variation, which arguably could comprise specimens at different ontogenetic stages. Although we did not carry out an independent assessment of ontogenetic stages, we carried out regressions within individual genera and species, as a preliminary assessment of potential ontogenetic trends. In most cases, these regressions did not provide statistical support to either confirm or discard a potential ontogenetic allometry (Additional file 1: Table S15), which is probably due to reduced sample sizes and/or non-ontogenetic variation. The exception was Coelophysis bauri, which presented a potential ontogenetic trend of positive allometry, but this trend was not significantly different from isometry when analyzing Coelophysis as a genus (C. bauri + C. rhodesiensis).

In non-theropod dinosaurs, studies of different ontogenetic stages are available for the ornithischian Psittacosaurus lujiatunensis [79] and for the basal sauropodomorphs Massospondylus carinatus [80], Riojasaurus incertus [81], and Mussaurus patagonicus [82]. In all of these non-theropod dinosaurs, early stages show greater forelimb proportions. For some of these, it has been suggested that early stages presented quadrupedal locomotion, and then transitioned into bipedal locomotion and proportionally shorter forelimbs $[79,80]$. Biomechanical studies of different ontogenetic stages of Mussaurus have now confirmed this quadrupedal-to-bipedal transition, showing how the center of mass was placed more anteriorly in younger individuals, and then shifted posteriorly as the tail grew proportionally larger and the neck became more slender [82]. The fact that the forelimbs experienced decreased growth along ontogeny was likely related to their decreased function in locomotion. The ontogenetic transition from quadrupedal hatchlings to bipedal adults has been suggested to be widespread among dinosaurs, and to represent the ancestral condition for this group [76]. In this regard, the absence of negative allometry in the evolution of Coelophysoidea (and possibly, in the ontogeny of Coelophysis) could be a derived condition. Alternatively, because Coelophysoidea is an outgroup to all other theropods in our analysis, it may represent the primitive trend for Theropoda. If so, negative allometry may have become the main trend shortly thereafter, in a slightly more exclusive clade approaching Averostra.

Many theropods could have presented an important ontogenetic phase of decreased forelimb growth, as suggested by the main evolutionary trend of negative allometry and the negative coefficients found in several subclades. Decreased forelimb growth in turn suggests decreased function along ontogeny. This decrease does not imply the absence of function: Most likely, some important function was performed at early stages, and was then lost in ontogeny, while other functions continued to be performed, such as those that have been welldiscussed for adults (especially the manipulation and carrying of prey, [83-86]). The proposed phase of decreased forelimb growth may have extended through a 
substantial portion of ontogeny, as suggested by ontogenetic data from Allosaurus [76], and the fact that evolutionary negative allometry is observable across a broad range of body sizes (including the upper large/gigantic range). However, it is hard to infer how early in ontogeny could the onset of decreased forelimb growth occur. Upon hatching, the forelimbs could have first experienced an early burst of positive allometric growth (as in modern birds), only experiencing decreased growth thereafter. Alternatively, forelimbs may have already been proportionally large, as in the quadrupedal hatchlings of non-theropod dinosaurs. Fossil evidence from theropod hatchlings is sparse, and mostly inferred from near-hatching embryos that cannot be confidently assigned to an adult species. In Therizinosauria, embryos have been described as having forelimb and hindlimb elements of similar size, along with the suggestion that hatchlings were quadrupedal [87]. Although no measurements were provided, such forelimb proportions would be greater than any adult members of Therizinosauria, supporting ontogenetic negative allometry in this clade, and large forelimb proportions upon hatching. In Oviraptorosauria, a clade that deviates from the main trend of negative allometry, the forelimb proportions of embryos resemble those of adult members of this clade [88] suggesting ontogenetic isometry, as expected from their evolutionary trend.

More discussion about the ontogeny of forelimb growth and locomotion is available for theropods that are closer to birds, especially in relation to wing assisted locomotion and the origin of flight. The recent description of a juvenile specimen of Deinonychus, including partial remains of the forelimbs, shows that forelimbs were proportionally larger at early stages, but also suggests that they were functionally different from adults [89]. The presence of a well-developed olecranon process only in the juvenile specimen has been argued to allow for greater extension of the arm (in contrast with a more permanently flexed position of the elbow in the adult), suggesting some form of wing assisted locomotion [89]. This is reasonable considering the presence of large remigial feathers on the forearms of Pennaraptora (including Dromaeosauridae), and the fact that these feathers were well developed even before hatching in closely related basal Aves [90, 91]. The case of Deinonychus may be compared with that of the modern bird Alectura lathami, where wing assisted locomotion is more developed in juveniles than adults [92, 93]. For theropod taxa close to Aves, wing-assisted locomotion (such as incline running, or even flight at early, small body sizes) provides an important function that could have reasonably been lost along ontogeny, as body size increased. It is worth noting that our data confirms that Dromaeosauridae has a marked evolutionary trend of negative allometry, that is significantly different from isometry (Table 2, supplementary Tables S5, S13 and S14). Thus, despite evolving small volant taxa such as Microraptor, there was no switch to positive allometry as the main evolutionary trend, unlike the closely related Aves [94].

Theropods leading to Aves experienced a marked decrease in body size $[6,7]$ which is consistent with an accompanying trend of paedomorphosis in skull morphology $[95,96]$. As such, besides traits such as an enlarged orbit and braincase, proportionally larger forelimbs could be another juvenile trait [10]. Forelimbs of paedomorphic adults would have remained functional, omitting the later stages of decreased function and negative allometric growth. In this regard, it is worth noting that both Ornithomimosauria and Oviraptorosauria show bird-like skulls with arguably paedomorphic traits, which also coincides with the loss of negative forelimb allometry as an evolutionary trend in these subclades (see the supplementary discussion in Additional file 1). An intriguing possibility is that in the lineage leading to birds, wing-assisted locomotion first evolved in juveniles with proportionally larger forelimbs, perhaps as a mechanism of predator avoidance, and only later became an adult trait in smaller paedomorphic forms. Hopefully, new fossil evidence will provide increasingly detailed information about forelimb ontogeny along the dinosaur-bird transition.

\section{Conclusions}

Our results confirm that negative forelimb allometry is the main evolutionary trend for non-avian theropods. Through Phylogenetic ANCOVA testing, we also identified that Coelophysoidea, Ornithomimosauria and Oviraptorosauria deviate from the main trend of negative allometry, with support for evolutionary trends closer to isometry.

Evolutionary allometric trends often reflect ontogenetic allometries, which suggests an important stage of negative allometric growth of the forelimb during the ontogeny of most non-avian theropods. Accordingly, a different ontogenetic trend can be expected for those subclades with evolutionary trends that deviate from negative forelimb allometry. In modern birds, allometric growth of the limbs is related to locomotor and behavioral changes along ontogeny. Fossil evidence supports slower growth of the forelimb during ontogeny as an ancestral condition for dinosaurs, likely related to bipedism and decreased forelimb use. In subclades closer to Aves, such as Dromeosauridae, early ontogenetic stages may have used their forelimbs in wing assisted-locomotion, that was lost at later ontogenetic stages, as body size increased. We propose that proportionally longer arms of juveniles became adult traits in the small-sized and paedomorphic Aves. 


\section{Supplementary information}

Supplementary information accompanies this paper at https://doi.org/10. 1186/s12983-019-0342-9.

Additional file 1. Supplementary Methods, Tables and Figures

Additional file 2. Dataset used for analysis

\section{Abbreviations}

BM: Brownian Motion; FL: Femoral Length; HL: Humeral Length

OLS: Ordinary Least Squares; OU: Ornstein-Uhlenbeck;

PANCOVA: Phylogenetic ANCOVA; PGLS: Phylogenetic Generalized Least

Squares; PIC: Phylogenetic Independent Contrasts

\section{Acknowledgements}

We thank our reviewers, whose constructive criticism that helped us to improve this publication. We thank Roberto Yury-Yañez for useful input in early stages of this work

\section{Authors' contributions}

Research idea was proposed by AV. JP and MM designed the methodology for the statistical analyses. JP and SSA compiled the dataset and constructed the base topologies. JP conducted the analyses. JP and AV discussed the results. JP wrote the first draft of the manuscript. JP and AV wrote the final version of the manuscript. All authors read and approved the final manuscript.

\section{Funding}

Financial funding was provided by Grants Anillo ACT 172099 and FONDECYT 1190891, CONICYT, Government of Chile.

\section{Availability of data and materials}

Dataset used in the present work is available in Additional file 2.

\section{Ethics approval and consent to participate}

Not applicable.

\section{Consent for publication}

Not applicable.

\section{Competing interests}

The authors declare that they have no competing interests.

\section{Author details}

'Laboratorio de Ontogenia y Filogenia, Departamento de Biología, Facultad de Ciencias, Universidad de Chile., Las Palmeras 3425, Santiago, Chile. 2Laboratorio de Genética y Evolución, Departamento de Ciencias Ecológicas, Facultad de Ciencias, Universidad de Chile., Las Palmeras 3425, Santiago, Chile.

Received: 17 July 2019 Accepted: 29 October 2019

Published online: 02 December 2019

\section{References}

1. Nopcsa BF. On the origin of flight in birds. Proc Zool Soc London. 1923;93: 463-77.

2. Bock WJ. The origin and radiation of birds*. Ann N Y Acad Sci. 1969;167: 147-55.

3. Ostrom JH. Bird flight: how did it begin? Am Sci. 1979:67:46-56.

4. Dial KP. Wing-assisted incline running and the evolution of flight. Science. 2003;299:402-4

5. Sullivan C, Xu X, O'Connor JK. Complexities and novelties in the early evolution of avian flight, as seen in the Mesozoic Yanliao and Jehol biotas of Northeast China. Palaeoworld. 2016:26:212-29.

6. Brusatte SL, Lloyd GT, Wang SC, Norell MA. Gradual assembly of avian body plan culminated in rapid rates of evolution across the dinosaur-bird transition. Curr Biol Elsevier Ltd. 2014;24:2386-92.

7. Lee MSY, Cau A, Naish D, Dyke GJ. Sustained miniaturization and anatomical innovation in the dinosaurian ancestors of birds. Science. 2014;345:562-6.

8. Chatterjee S, Templin RJ. Feathered coelurosaurs from China: new light on the arboreal origin of avain flight. In: Currie PJ, Koppelhus EB, Shugar MA,
Wright $\mathrm{J}$, editors. Feather dragons stud transit from dinosaur to birds. Bloomington: Indiana University Press; 2004. p. 251-80.

9. Xu X, You H, Du K, Han F. An archaeopteryx-like theropod from China and the origin of Avialae. Nature Nature Publishing Group. 2011:475:465-70.

10. Vargas AO. Evolution of arm size in theropod dinosaurs: a developmental hypothesis. Not Mens Mus Nac Hist Nat. 1999; 338:16-9. Available from: http://publicaciones.mnhn.gob.cl/668/w3-article-66661.html.

11. Dececchi TA, Larsson HCE. Body and limb size dissociation at the origin of birds: uncoupling Allometric constraints across a macroevolutionary transition. Evolution. 2013;67:2741-52

12. Nudds RL. Wing-bone length allometry in birds. J Avian Biol. 2007;38:515-9.

13. Rohlf FJ. A comment on phylogenetic correction. Evolution. 2006;60:1509-15.

14. Zhang F, Zhou Z, Xu X, Wang $X$, Sullivan C. A bizarre Jurassic maniraptoran from China with elongate ribbon-like feathers. Nature. 2008;455:1105-8.

15. Pei R, Li Q, Meng Q, Norell MA, Gao K-Q. New specimens of Anchiornis huxleyi (Theropoda: Paraves) from the late Jurassic of northeastern China. Bull Am Museum Nat Hist. 2017:411:1-67.

16. Senter P. A new look at the phylogeny of coelurosauria (Dinosauria: Theropoda). J Syst Palaeontol. 2007:5:429-63.

17. Agnolin F, Novas FE. Avian ancestors. Springer. Dordrecht: Springer Netherlands; 2013.

18. Hartman S, Mortimer M, Wahl WR, Lomax DR, Lippincott J, Lovelace DM. A new paravian dinosaur from the late Jurassic of North America supports a late acquisition of avian flight. Peer J. 2019;7:e7247.

19. Wang M, O'Connor JK, Xu X, Zhou Z. A new Jurassic scansoriopterygid and the loss of membranous wings in theropod dinosaurs. Nature Springer US 2019:569:256-9.

20. Carrano MT. Body-size evolution in the Dinosauria. In: Carrano MT, Gaudin TJ, Blob RW, Wible JR, editors. Amniote paleobiology: perspectives on the evolution of mammals, birds, and reptiles. Chicago: University of Chicago Press; 2006. p. 225-68.

21. Sookias RB, Butler RJ, Benson RBJ. Rise of dinosaurs reveals major body-size transitions are driven by passive processes of trait evolution. Proc $R$ Soc $B$ Biol Sci. 2012;279:2180-7.

22. Hedrick BP, Manning PL, Lynch ER, Cordero SA, Dodson P. The geometry of taking flight: limb morphometrics in Mesozoic theropods. J Morphol. 2015; 276:152-66

23. Middleton KM, Gatesy SM. Theropod forelimb design and evolution. Zool J Linn Soc. 2000;128:149-87.

24. Garamszegi LZ. Uncertainties due to within-species variation in comparative studies: measurement errors and statistical weights. In: Garamszegi LZ, editor. Modern Phylogenetic Comparative Methods and Their Application in Evolutionary Biology. Berlin, Heidelberg: Springer Berlin; 2014. p. 157-99.

25. Hone DWE, Farke AA, Wedel MJ. Ontogeny and the fossil record: what, if anything, is an adult dinosaur? Biol Lett. 2016;12:20150947.

26. Huelsenbeck JP. Accommodating phylogenetic uncertainty in evolutionary studies. Science. 2000:288:2349-50.

27. Pagel M, Lutzoni F. Accounting for phylogenetic uncertainty in comparative studies of evolution and adaptation. In: Lässig M, Valleriani A, editors. Biological Evolution and Statistical Physics. Berlin, Heidelberg: Springer Berlin; 2002. p. 148-61.

28. Felsenstein J. Inferring Phylogenies. Sunderland: Sinauer Associates; 2004.

29. Rangel TF, Colwell RK, Graves GR, Fučíková K, Rahbek C, Diniz-Filho JAF. Phylogenetic uncertainty revisited: implications for ecological analyses. Evolution. 2015:69:1301-12

30. Maddison WP, Maddison DR. Mesquite: a modular system for evolutionary analysis; 2017.

31. Benson RBJ, Carrano MT, Brusatte SL. A new clade of archaic large-bodied predatory dinosaurs (Theropoda: Allosauroidea) that survived to the latest Mesozoic. Naturwissenschaften. 2010;97:71-8.

32. Novas FE, Agnolín FL, Ezcurra MD, Porfiri J, Canale Jl. Evolution of the carnivorous dinosaurs during the cretaceous: the evidence from Patagonia. Cretac Res. 2013;45:174-215

33. Porfiri JD, Novas FE, Calvo JO, Agnolín FL, Ezcurra MD, Cerda IA. Juvenile specimen of Megaraptor (Dinosauria, Theropoda) sheds light about tyrannosauroid radiation. Cretac Res. 2014:51:35-55.

34. RStudio Team. RStudio: integrated development environment for R. Boston; 2016. https://support.rstudio.com/hc/en-us/articles/206212048Citing-RStudio.

35. R Core Team. R: A Language and Environment for Statistical Computing. Vienna; 2017. https://cran.r-project.org/doc/FAQ/R-FAQ.htm|\#Citing-R. 
36. Paradis E, Claude J, Strimmer K. APE: analyses of Phylogenetics and evolution in R language. Bioinformatics. 2004;20:289-90.

37. Bapst DW. Paleotree : an R package for paleontological and phylogenetic analyses of evolution. Methods Ecol Evol. 2012;3:803-7.

38. Bapst DW. A stochastic rate-calibrated method for time-scaling phylogenies of fossil taxa. Methods Ecol Evol. 2013;4:724-33.

39. Bapst DW, Hopkins MJ. Comparing cal3 and other a posteriori time-scaling approaches in a case study with the pterocephaliid trilobites. Paleobiology. 2017:43:49-67.

40. Starrfelt J, Liow LH. How many dinosaur species were there? Fossil bias and true richness estimated using a Poisson sampling model. Philos Trans R Soc B Biol Sci. 2016:371:20150219.

41. Figueirido B, Martín-Serra A, Tseng ZJ, Janis CM. Habitat changes and changing predatory habits in north American fossil canids. Nat Commun. 2015;6:7976.

42. Firmat C, Lozano-Fernández I, Agustí J, Bolstad GH, Cuenca-Bescós G, Hansen TF, et al. Walk the line: 600000 years of molar evolution constrained by allometry in the fossil rodent Mimomys savini. Philos Trans R Soc B Biol Sci. 2014;369:20140057

43. Hone DWE, Dyke GJ, Haden M, Benton MJ. Body size evolution in Mesozoic birds. J Evol Biol. 2008;21:618-24.

44. Felsenstein J. Comparative methods with sampling error and within-species variation: contrasts revisited and revised. Am Nat. 2008;171:713-25.

45. Blomberg SP, Lefevre JG, Wells JA, Waterhouse M. Independent contrasts and PGLS regression estimators are equivalent. Syst Biol. 2012;61:382-91.

46. Revell $L$. Size-correction and principal components for interspecific comparative studies. Evolution. 2009;63:3258-68.

47. Symonds MRE, Blomberg SP. A primer on phylogenetic generalised least squares. In: Garamszegi LZ, editor. Modern phylogenetic comparative methods and their application in evolutionary biology. Berlin, Heidelberg: Springer Berlin Heidelberg; 2014. p. 105-30.

48. Grafen A. The phylogenetic regression. Philos Trans R Soc B Biol Sci. 1989; 326:119-57.

49. Felsenstein J. Maximum-likelihood estimation of evolutionary trees from continuous characters. Am J Hum Genet. 1973;25:471-92.

50. Felsenstein J. Phylogenies and the comparative method. Am Nat. 1985;125:1-15.

51. Hansen TF. Stabilizing selection and the comparative analysis of adaptation. Evolution. 1997:51:1341.

52. Cooper N, Thomas GH, Venditti C, Meade A, Freckleton RP. A cautionary note on the use of Ornstein Uhlenbeck models in macroevolutionary studies. Biol J Linn Soc. 2016;118:64-77.

53. Pagel M. Inferring the historical patterns of biological evolution. Nature. 1999;401:877-84.

54. Münkemüller T, Lavergne S, Bzeznik B, Dray S, Jombart T, Schiffers K, et al. How to measure and test phylogenetic signal. Methods Ecol Evol. 2012;3: 743-56.

55. Pinheiro J, Bates D, DebRoy S, Sarkar D, R Core Team. nlme: Linear and nonlinear mixed effects models. 2017.

56. Rubin DB. Multiple imputation for nonresponse. In: Rubin DB, editor. Surveys. Hoboken: Wiley; 1987.

57. Buuren S. Flexible imputation of missing data. Boca Ratón: Chapman and Hall/CRC; 2012.

58. Martins EP. Conducting phylogenetic comparative studies when the phylogeny is not known. Evolution. 1996;50:12-22.

59. van Buuren S, Groothuis-Oudshoorn K. mice : Multivariate Imputation by Chained Equations in R. J Stat Softw. 2011:45:1-67.

60. Smaers JB, Rohlf FJ. Testing species' deviation from allometric predictions using the phylogenetic regression. Evolution. 2016;70:1145-9.

61. Poole W, Gibbs DL, Shmulevich I, Bernard B, Knijnenburg TA. Combining dependent P- values with an empirical adaptation of Brown's method. Bioinformatics. 2016;32:1430-6.

62. Senter $P$. Function in the stunted forelimbs of Mononykus olecranus (Theropoda), a dinosaurian anteater. Paleobiology. 2005;31:373-81.

63. Lü J, Currie PJ, Xu L, Zhang X, Pu H, Jia S. Chicken-sized oviraptorid dinosaurs from Central China and their ontogenetic implications. Naturwissenschaften. 2013;100:165-75.

64. Gould SJ. Allometry and size in ontogeny and phylogeny. Biol Rev. 1966;41: 587-638.

65. Alberch P, Gould S, Oster G, Wake D. Size and shape in ontogeny and phylogeny. Paleobiology. 1979;5:296-317.
66. Larson PM. Ontogeny, phylogeny, and morphology in anuran larvae: morphometric analysis of cranial development and evolution in Rana tadpoles (Anura: Ranidae). J Morphol. 2005;264:34-52.

67. Heers AM, Dial KP. Wings versus legs in the avian bauplan: development and evolution of alternative locomotor strategies. Evolution. 2015;69:305-20.

68. Faux C, Field DJ. Distinct developmental pathways underlie independent losses of flight in ratites. Biol Lett. 2017;13:20170234.

69. Ricklefs RE, White S, Cullen J. Postnatal development of Leach's stormpetrel. Auk. 1980;97:768-81.

70. Carrier D, Leon LR. Skeletal growth and function in the California gull ( Larus californicus ). J Zool. 1990;222:375-89.

71. Bennett MB. Post-hatching growth and development of the pectoral and pelvic limbs in the black noddy, Anous minutus. Comp Biochem Physiol - A Mol Integr Physiol. 2008;150:159-68.

72. Dial TR, Carrier DR. Precocial hindlimbs and altricial forelimbs: partitioning ontogenetic strategies in mallards (Anas platyrhynchos). J Exp Biol. 2012;215: 3703-10.

73. Galbraith $\mathrm{H}$. Adaptation and constraint in the growth pattern of lapwing Vanellus vanellus chicks. J Zool. 1988;215:537-48.

74. Gould SJ, Lewontin RC. The spandrels of san Marco and the Panglossian paradigm: a critique of the Adaptationist Programme. Proc R Soc B Biol Sci. 1979;205:581-98.

75. Lockley $M$, Kuhihara $R$, Mitchell L. Why tyrannosaurus rex had puny arms: an integral morphodynamic solution to a simple puzzle in theropod paleobiology. In: Larson P, Carpenter K, editors. Tyrannosaurus rex, Tyrant King. Bloomington: Indiana University Press; 2008. p. 130-64.

76. Bybee PJ, Lee AH, Lamm E-T. Sizing the Jurassic theropod dinosaur Allosaurus: assessing growth strategy and evolution of ontogenetic scaling of limbs. J Morphol. 2006;267:347-59.

77. Currie PJ. Allometric growth in tyrannosaurids (Dinosauria: Theropoda) from the upper cretaceous of North America and Asia. Can J Earth Sci. 2003;40: 651-65.

78. Larson P. The case for Nanotyrannus. In: Parrish JM, Molnar RE, Currie PJ, Koppelhus EB, editors. Tyrannosaurid Paelobiology. Bloomington: Indiana University Press; 2013. p. 14-53.

79. Zhao Q, Benton MJ, Sullivan C, Martin Sander P, Xu X. Histology and postural change during the growth of the ceratopsian dinosaur Psittacosaurus lujiatunensis. Nat Commun. Nature Publishing Group. 2013:4:1-8.

80. Reisz RR, Scott D, Sues H-D, Evans DC, Raath MA. Embryos of an early Jurassic prosauropod dinosaur and their evolutionary significance. Science. 2005:309:761-4.

81. Kilbourne BM, Makovicky PJ. Limb bone allometry during postnatal ontogeny in non-avian dinosaurs. J Anat. 2010;217:135-52.

82. Otero A, Cuff AR, Allen V, Sumner-Rooney L, Pol D, Hutchinson JR. Ontogenetic changes in the body plan of the sauropodomorph dinosaur Mussaurus patagonicus reveal shifts of locomotor stance during growth. Sci Rep. Springer US. 2019;9:7614.

83. Senter $\mathrm{P}$, Robins $\mathrm{JH}$. Range of motion in the forelimb of the theropod dinosaur Acrocanthosaurus atokensis, and implications for predatory behaviour. J Zool. 2005:266:307-18.

84. Senter P. Comparison of forelimb function between Deinonychus and Bambiraptor (Theropoda: Dromaeosauridae). J Vertebr Paleontol. 2006;26: 897-906.

85. Hutchinson JR, Allen $\mathrm{V}$. The evolutionary continuum of limb function from early theropods to birds. Naturwissenschaften. 2009;96:423-48.

86. Xu X, Tan Q, Sullivan C, Han F, Xiao D. A short-armed troodontid dinosaur from the upper cretaceous of inner Mongolia and its implications for troodontid evolution. Farke AA, editor. PLoS One. 2011;6:e22916.

87. Kundrát M, Cruickshank ARI, Manning TW, Nudds J. Embryos of therizinosauroid theropods from the upper cretaceous of China: diagnosis and analysis of ossification patterns. Acta Zool. 2008:89:231-51.

88. Weishampel DB, Fastovsky DE, Watabe M, Varricchio D, Jackson F, Tsogtbaatar K, et al. New oviraptorid embryos from Bugin-Tsav, Nemegt formation (upper cretaceous), Mongolia, with insights into their habitat and growth. J Vertebr Paleontol. 2008;28:1110-9.

89. Parsons WL, Parsons KM. Morphological Variations within the Ontogeny of Deinonychus antirrhopus (Theropoda, Dromaeosauridae). Dodson P, editor. PLoS One. 2015:10:e0121476. 
90. Zhou Z, Zhang F. A Precocial avian embryo from the lower cretaceous of China. Science. 2004;306:653.

91. Chinsamy A, Elzanowski A. Evolution of growth pattern in birds. Nature. 2001;412:402-3.

92. Dial KP, Jackson BE. When hatchlings outperform adults: Locomotor development in Australian brush turkeys (Alectura lathami, Galliformes). Proc R Soc B Biol Sci. 2011;278:1610-6.

93. Dececchi TA, Larsson HCE, Habib MB. The wings before the bird: an evaluation of flapping-based locomotory hypotheses in bird antecedents. PeerJ. 2016;4:e2159.

94. Yury-Yáñez RE, Soto-Acuña S, Vargas AO. Negative allometry of forelimb size in non- ornithurine Paraves. Ameghiniana. 2009;46:55-6.

95. Thulborn RA. Birds as neotenous dinosaurs. Christchurch: Rec. New Zeal. Geol. Surv; 1985. p. 90-2.

96. Bhullar B-AS, Marugán-Lobón J, Racimo F, Bever GS, Rowe TB, Norell MA, et al. Birds have paedomorphic dinosaur skulls. Nature. 2012;487:223-6.

\section{Publisher's Note}

Springer Nature remains neutral with regard to jurisdictional claims in published maps and institutional affiliations.

Ready to submit your research? Choose BMC and benefit from:

- fast, convenient online submission

- thorough peer review by experienced researchers in your field

- rapid publication on acceptance

- support for research data, including large and complex data types

- gold Open Access which fosters wider collaboration and increased citations

- maximum visibility for your research: over $100 \mathrm{M}$ website views per year

At BMC, research is always in progress.

Learn more biomedcentral.com/submissions 